\title{
A novel minimally invasive presacral approach and instrumentation technique for anterior L5-S1 intervertebral discectomy and fusion
}

\author{
Technical note and case presentations
}

\author{
Nicola Marotta, M.D., Murat Cosar, M.D., Luiz Pimenta, M.D., \\ AND LARRY T. KHOO, M.D. \\ Division of Neurosurgery, University of California at Los Angeles, California; Department of \\ Neurosciences, Neurosurgery, University of Rome "La Sapienza," Rome, Italy; Division of \\ Neurosurgery, Okmeydani Education Hospital, Istanbul, Turkey; and Department of Neurosurgery, \\ Santa Rita Hospital, Sao Paolo, Brazil
}

\begin{abstract}
Object. The authors describe a new paracoccygeal approach to the L5-S1 junction for interbody fusion with transsacral instrumentation. The purpose of this technical note is to demonstrate a novel surgical approach, technique, and instrumentation system for the treatment of L5-S1 instability in degenerative disc disease and spondylolisthesis.

Methods. This technical note highlights the AxiaLif (TranS1) transsacral system as an alternative method to transforaminal lumbar interbody fusion or posterior lumbar interbody fusion. Via a novel presacral approach corridor, a truly percutaneous L5-S1 discectomy, interbody distraction, and fixation are achieved, and retroperitoneal viscera and dorsal neural elements are avoided. Percutaneous pedicle screw fixation is then used to provide additional stabilization at the treated level.

Conclusions. This novel technique of interbody distraction and fusion via a truly percutaneous approach corridor allows for circumferential treatment of the lower lumbar segments with minimal risk to the anterior organs and dorsal neural elements.
\end{abstract}

\section{KEY WORDS • instrumentation • interbody fusion • paracoccygeal approach • transsacral instrumentation}

Symptomatic instability and stenosis of the lower L5-S1 and L4-5 vertebral segments are very common. Generally, anterior and posterior approaches are chosen for direct exposure of the lumbosacral spine. These kinds of open approaches are often poorly tolerated by patients because they require muscular and ligamentous dissection, neural retraction, and anular disruption, sometimes with mobilization of vascular and visceral structures. In addition to these potential problems, vascular injury, sympathetic dysfunction, bowel injury, and neurological deficit often also complicate the perioperative and surgical course..$^{15,16}$

More recently, technological advances in which small incisions and portals are used have allowed surgeons to perform L5-S1 fusion via posterolateral or anterior approaches through less invasive techniques. ${ }^{1,3-13,17,19}$ The AxiaLif system (TranS1, Inc., Wilmington, NC) combines the advantages of minimally invasive spinal surgical techniques with a novel corridor of approach. Via a small para-

Abbreviations used in this paper: $\mathrm{AP}=$ anteroposterior; $\mathrm{MR}=$ magnetic resonance; $\mathrm{VB}=$ vertebral body $3 \mathrm{D}=$ three-dimensional. coccygeal incision, a presacral approach corridor is percutaneously developed for access to the anterior lumbosacral body and, subsequently, to the L5-S1 intervertebral space, while preserving the integrity of the muscles, ligaments, and disc anulus. ${ }^{2,3}$ In this technical note, we describe the minimally invasive surgical technique of this presacral approach to the anterior lumbosacral body for L5-S1 interbody fusion.

\section{OPERATIVE TECHNIQUE}

After induction of general anesthesia, the patient is positioned prone on a Jackson or other compatible radiolucent operative table (Fig. 1A). A 20-French catheter is optionally inserted into the rectum, and the balloon is insufflatedwith $10 \mathrm{ml}$ air to provide improved visualization of the rectum during lateral fluoroscopy. The anus is isolated with an occlusive dressing that is placed to separate it from the paracoccygeal working area, which is more dorsal. Optional intraoperative electromyography and/or somatosensory evoked potential neurophysiological monitoring can be used during the procedure for surveillance of neural integri- 
ty during decompression, interbody distraction, and screw placement. A fluoroscopic C-arm is then brought into the surgical field to provide for real-time lateral and AP imaging during the entire procedure. The sacrococcygeal region and the gluteal zone are washed and draped in the usual sterile fashion.

A $15-\mathrm{mm}$ incision is marked on the skin $20 \mathrm{~mm}$ caudal to the left or right paracoccygeal notch (Fig. 1B). After a local anesthetic containing 25\% Marcaine with 1:200,000 epinephrine has been applied to the area, a No. 15 blade is used to incise the skin and underlying fascia. Blunt finger dissection is passed through the opening to ensure that the fascia is appropriately opened.

The guide pin introducer/stylet assembly is inserted into the incision and slowly and gently advanced along the anterior midline of the sacrum. Direct tactile feedback is obtained from the stylet to ensure continuous bone contact of the introducer tip. A rhythmic small-amplitude oscillating movement is used to sweep the presacral fat and anterior contents away from the osseous floor of the pelvis (Fig. 1C). This maneuver is completed using frequent biplanar fluoroscopic control to ensure that the stylet tip remains in the midline, thus avoiding inadvertent entry into the paramedially located sacral foramen along the anterior sacral surface. The guide pin introducer is ultimately engaged on the anterior cortex of the S1-2 junction under fluoroscopic guidance.

After preliminary contact with the inferior aspect of the S1-2 junction, biplanar fluoroscopy is used to plan a trajectory through the sacrum, L5-S1 disc space, and L-5 VB that will ultimately be the final path for placement of the AxiaLif threaded cage. Adjustments of the stylet are made such that this trajectory will pass through the middle and anterior portions of the L5-S1 intervertebral disc space while still ending in the anterior column of the L-5 VB. In the AP plane, it is similarly important to ensure that the ultimate transdiscal trajectory will be near the midline and not too far off laterally in either direction. This planning step can be facilitated by placing an unsterile Steinmann pin directly on the fluoroscopic monitor.

Once the optimal trajectory has been obtained, the blunt guide introducer is exchanged for the sharp guide pin on a handle and then docked at the S1-2 junction.

After the trajectory is again fluoroscopically reconfirmed, a cannulated slap hammer is used to tap the guide pin trough the sacrum (Fig. 1D) along the planned vector into the L5-S1 disc space. At this point, the guide pin and handle are removed and a guide pin extension is attached to permit the placement of a series of dilators, which will dilate the presacral soft tissue and create the osseous working channel in the sacrum itself. The first beveled 6-mm tubular dilator is then advanced over the guide pin using the slap hammer, with the bevel facing ventrally to facilitate positioning of the visceral contents anteriorly and away. On contact with the sacrum, the dilator is rotated $180^{\circ}$ to match the bevel with the inclination of the sacrum. It is then driven directly through the sacral surface and countersunk several millimeters to firmly seat it within the bone. This dilator is then removed and the same series of steps is repeated with a larger $(8-\mathrm{mm})$ dilator. After removal of the 8-mm dilator, a $10-\mathrm{mm}$ dilator is inserted and assembled with a thin-walled dilator sheath that is slid over the $10-\mathrm{mm}$ dilator body. This combined assembly is advanced and anchored firmly in the sacrum using the slap hammer. Prior to final docking, biplanar fluoroscopic confirmation is critical to ensure that the optimal trajectory has been maintained throughout the dilation and docking procedures. The guide pin and dilator tubes are removed, thereby establishing a secure presacral-transsacral working corridor for subsequent intervertebral access, discectomy, reaming, distraction, and threaded cage placement.

A 9-mm threaded reamer is advanced down the working portal into the sacrum to create the osseous channel into the L5-S1 disc space, using biplanar fluoroscopy to continuously monitor the position of the drill tip. As the reamer is gradually rotated, it is passed through the superior S-1 endplate and the L5-S1 disc space to come to rest lightly on the inferior endplate of L-5 without penetrating it. The 9-mm reamer is then withdrawn, with the bone contained in the threads saved for later use as autologous bone graft material.

Next the surgeon performs a partial volumetric discectomy, using a variety of propietary cutting-loop devices and disc extractors that were specifically designed for a transsacral approach (Fig. 1E). These devices are composed of cutting loops made from Nitinol memory metal that allows for introduction through a working sheath in a straight coaxial fashion. Once it is placed beyond the confines of the introducer sheath, however, the loop will return to a preset angle and curvature to allow for a variety of cutting angles and lengths. These cutting loops are passed into the L5-S1 disc space sequentially under fluoroscopic guidance to complete a rotational-type discectomy. In addition, forward- and back-angled loops are inserted and rotated under manual pressure to carve off the cartilaginous endplates and create some bleeding cortical surfaces for arthrodesis. After the introduction of each cutting loop, a special wire brush-type capture device is inserted, implanted, and rotated to capture the fragmented disc material. In this fashion, a sizable radial removal of the disc nucleus is achieved while outer anular integrity is maintained.

After volumetric discectomy has been completed, a funnel-type cannula is inserted into the L5-S1 disc space for the purpose of introducing the bone graft material. Typically, the graft material is composed of a combination of autologous bone obtained during reaming; iliac crest bone marrow aspirate, with or without concentration into a carrier matrix; demineralized bone matrices; and calcium triphosphates. Optionally, bone morphogenetic protein (such as Infuse or OP-1) can also be introduced as graft material into the disc space. Through the funnel, approximately 10 to $15 \mathrm{ml}$ of graft material is then packed directly into the interbody space while the beveled end of the funnel is rotated (Fig. 2A).

After interbody arthrodesis, a 7.5-mm-diameter drill is inserted through the working sheath and intervertebral space, penetrating directly into the L-5 VB under biplanar fluoroscopic guidance (Fig. 2B). Once the trajectory has been reconfirmed, the $7.5-\mathrm{mm}$ bit is rotated and advanced to within $1 \mathrm{~cm}$ of the superior L-5 endplate using frequent fluoroscopic visualization. This reamer is then removed, and the bone can be reintroduced into the disc space for graft material.

A final guide pin is inserted through the dilator sheath, which is then removed. A larger introducer cannula is inserted over the final guide pin to permit placement of the $3 \mathrm{D}$ axial rod prosthetic device. This titanium prosthesis is 

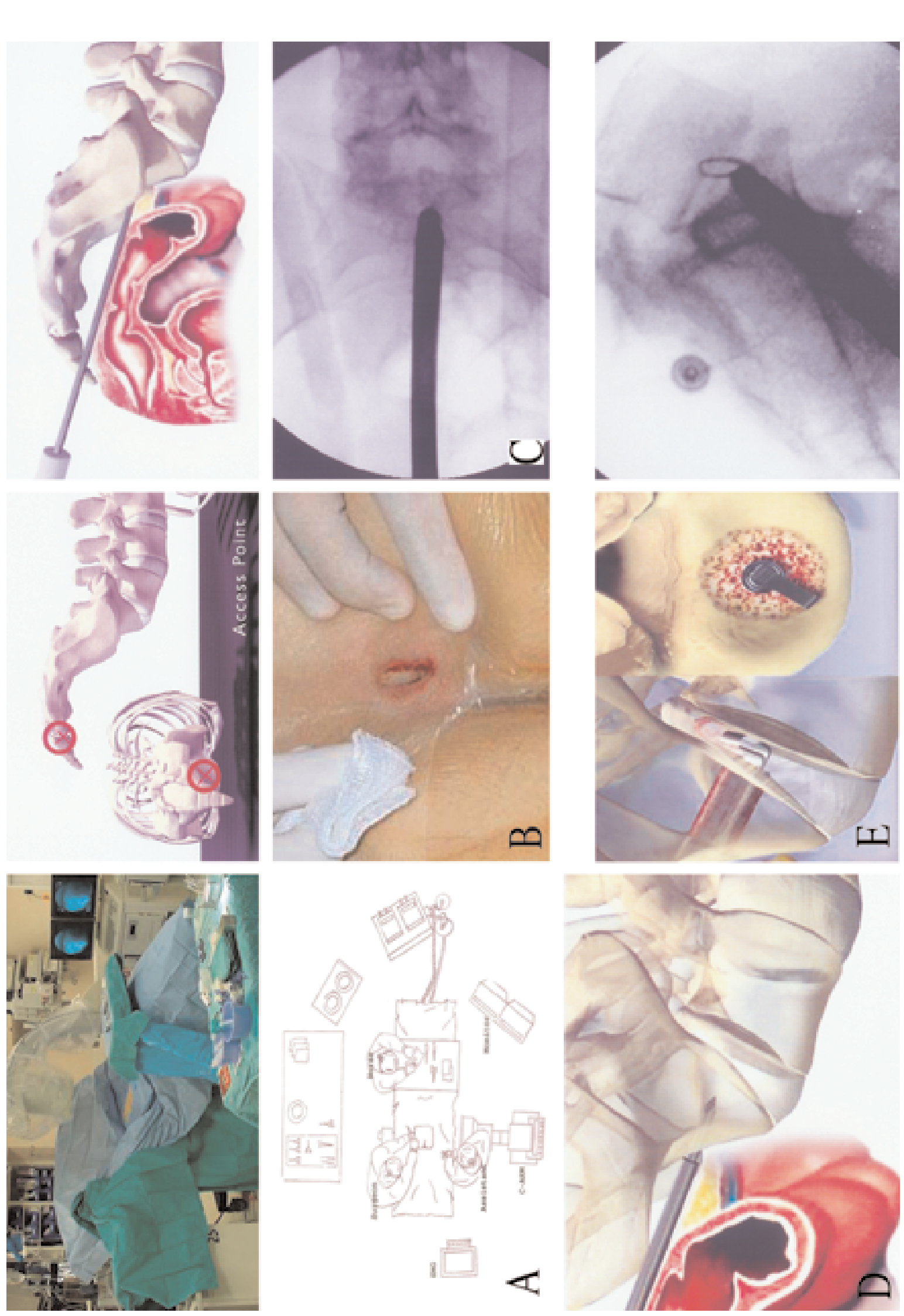

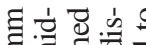

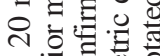

은 일

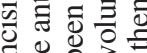

$\exists D \geq 7$

일

क 휴 흉

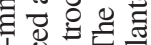

늘 월

\&

60 0 ㅂ.

웡

包论

으을

춘

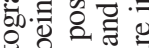

층혀

है

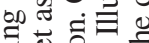

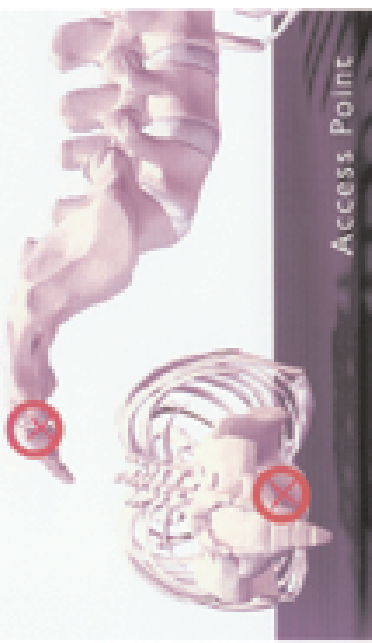

党.

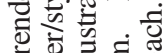

要焉

해월

घ.छ

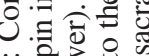

๑

해응

of

$0,40 .=$

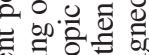

प्र.

낸 응 웡

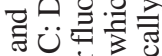

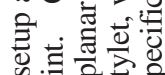

1

웝 웜

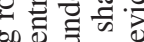

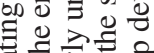

동 굴

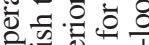

政

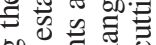

on 0 원

등

के $\frac{0}{2} \cdot \frac{0}{10}$

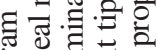

on

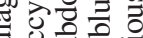

웡워

㯊

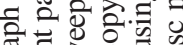

क.

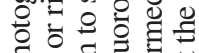

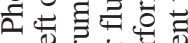

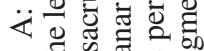

-

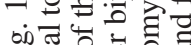

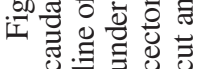



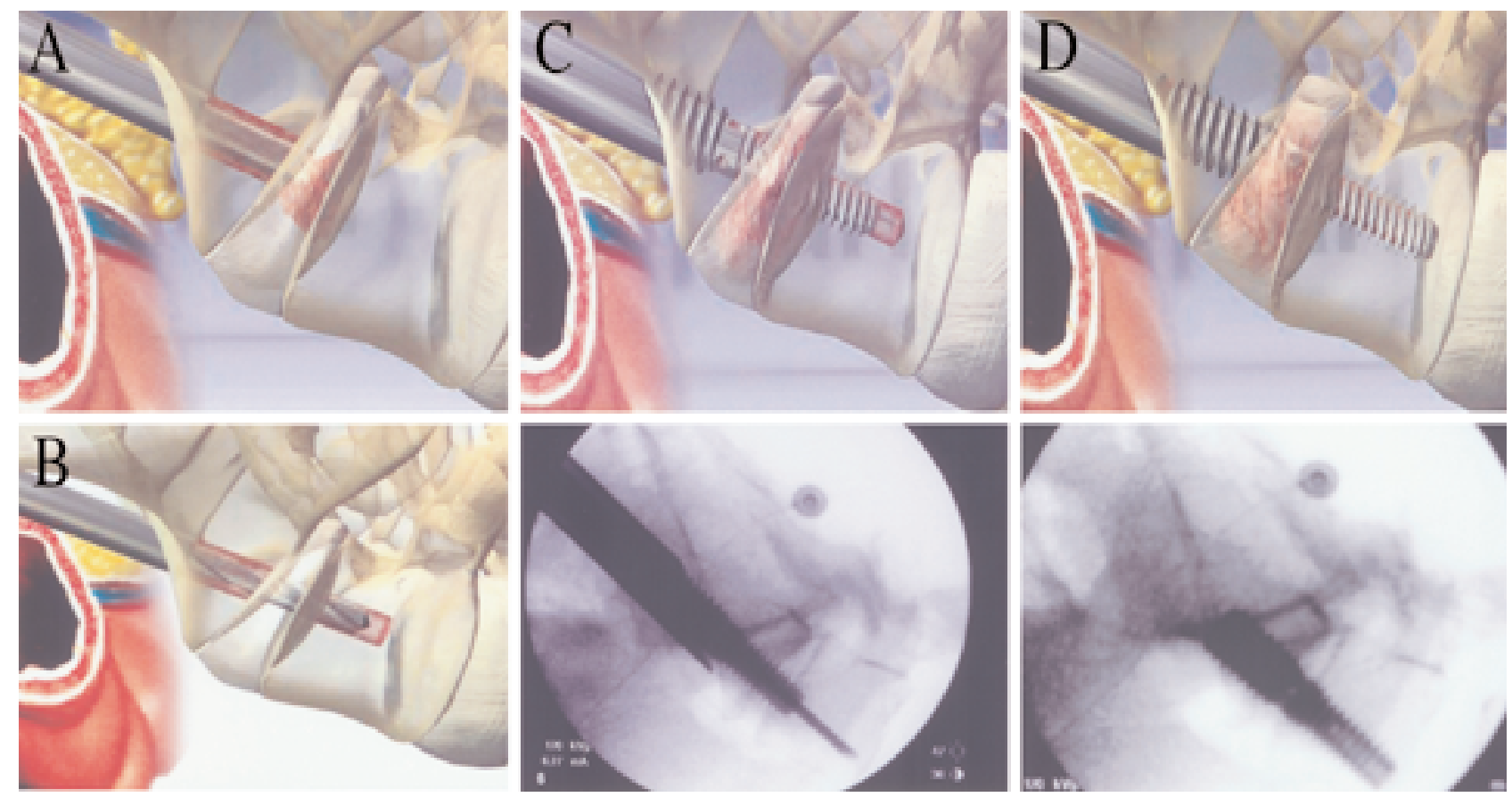

Fig. 2. Illustrations and fluoroscopic images. A: A funnel-type cannula is inserted into the L5-S1 disc space to introduce the bone graft material for arthrodesis, which is delivered by gradually rotating the beveled outlet to distribute the graft material evenly into the radial cavity. B: A 7.5-mm-diameter drill is inserted through the working sheath and intervertebral space to penetrate directly into the L-5 VB. C: The AxiaLif cage is inserted over the guide pin and engaged into the L-5 VB along the predetermined trajectory. D: After the prosthesis has been fully threaded into the L-5 VB, the differential screw pitch mechanism of the device creates distraction across the L5-S1 interbody space, thereby restoring lordosis and increasing foraminal height, as confirmed on lateral fluoroscopy (lower).

specially fabricated such that the threaded rod has two portions with different thread pitches. The superior aspect of the threaded cage that engages the L-5 VB has a diameter of $11 \mathrm{~mm}$ and a wider thread pitch compared with the inferior S-1 portion of the device, which has a diameter of 14 $\mathrm{mm}$ and a narrower thread pitch. The differential diameter and thread pitch result in a reverse lag-type screw action, leading to distraction of the intervertebral disc space height as it is threaded between the S-1 and L-5 VBs. Appropriate sizing is obtained by measuring the distance from the guide pin and by adding the amount of desired segmental distraction.

Once the appropriately sized 3D axial rod has been obtained, it is then cautiously inserted with a gentle movement over the previously introduced guide pin. Using a Thandle, it is gradually rotated through the sacrum and into the intervertebral space (Fig. 2C). As it enters the L-5 VB, the differential pitch creates the distraction mechanism across the disc space, which is confirmed under lateral fluoroscopic control (Fig. 2D). The rod is rotated until it is firmly seated under the previously created passage under the superior endplate of L-5. The guide pin is then removed. A tubular injection portal can then be docked on the inferior portion of the 3D axial rod to allow for additional injection of iliac crest bone marrow aspirate, bone morphogenetic protein, or other material, as the rod has a center channel with exterior ports to allow passage of the material into the intervertebral space. The injection portal is then disengaged, and a threaded plug is inserted to seal the rod to prevent graft extrusion.

The rod introducer cannula is gently withdrawn, and the working incision is copiously irrigated with antibioticimpregnated solution. The subcutaneous tissue is closed in an inverted manner, and DermaBond liquid dressing is applied in lieu of staples to faciliate sealing of the wound against liquid contamination for the next 7 days.

After anterior L5-S1 interbody fusion has been performed via the paracoccygeal approach, an L5-S1 minimally invasive screw fixation is undertaken. The L-5 and S-1 pedicles are localized with an 11-gauge bone needle under AP and lateral fluoroscopic guidance. Two small skin incisions are marked, and the fascia is incised. Through the muscles, a No. 11 Jamshidi needle is placed at the top of the pedicle and advanced into the pedicle. After biplanar fluoroscopic confirmation of the needle position has been obtained, the trocar is removed and exchanged for a Kirschner wire. After the sequential dilators have been placed, the pedicle is tapped and an appropriately sized cannulated screw and extender sleeve (Pathfinder; Abbott Spine, Austin, TX) are placed into the pedicle and VB under fluoroscopic control. The same steps are repeated for the placement of the other screws. An appropriately sized rod is then placed down the minimally invasive Pathfinder extender sleeves and locked into place. The sleeves are removed, and the small incisions are closed primarily with 0 Vicryl in the fascia and 3-0 Vicryl subcutaneously. Final 
biplanar confirmation of the construct is then obtained (Fig. 3).

\section{ILLUSTRATIVE CASES}

\section{Case 1}

This 36-year-old woman had a history of low-back degenerative disease and had experienced pain at L5-S1 for 14 years. The pain had increased in intensity during the past 6 months. She had undergone an L5-S1 discectomy 2 years previously for L5-S1 radiculopathy and had experienced relief of her pain that lasted 6 weeks. A comparison of an MR imaging study with a previous one demonstrated degenerative changes at the L5-S1 level, with significant desiccation of the disc and reduction in the height of the intervertebral space at this level (Fig. 4 upper). The patient underwent surgery with L5-S1 interbody fusion in which the AxiaLif transsacral system combined with L5-S1 minimally invasive Pathfinder screw fixation was used (Fig. 4 lower). During the procedure intraoperative electromyographic and somatosensory evoked potential neurophysiological monitoring were used, which demonstrated no change. The duration of surgery was 90 minutes. The postoperative course was uneventful, and the duration of hospital stay was 40 hours. At the time of discharge, the patient had complaints only of incisional pain, with no further radiculopathy.

\section{Case 2}

This 50-year-old woman suffered a fall several years prior to presentation and experienced brief L5-S1 sciatica that resolved early on. Over time, progressive severe lumbago developed and was characterized by intermittent episodes of bilateral symptoms at L-5. Serial MR imaging and x-ray studies revealed diminution of the L5-S1 intervertebral height with significant loss of foraminal height and secondary L-5 root entrapment (Fig. 5 upper). The patient underwent surgery with L5-S1 interbody fusion in which the AxiaLif transsacral system combined with L5-S1 minimally invasive Pathfinder screw fixation was used. The duration of surgery was 1 hour and 45 minutes, with a blood loss of less than $50 \mathrm{ml}$. The patient was discharged within 32 hours with only mild to moderate pain. Early 9-month lateral x-ray films revealed no subsidence of the segment, with bridging bone seen across the disc space and no hardware migration (Fig. 5 lower).

\section{DISCUSSION}

Degenerative disease of the disc space frequently affects the anterior column of the lower lumbosacral spine, as normal loading of the lumbar spine directs more than $80 \%$ of the axial load over the anterior column in the lower levels. As such, fusion for treatment of spinal instability and back pain at the L4-5 and L5-S1 segments is extremely common. The open anterior and posterior surgical approaches to the lumbosacral spine often cause significant muscular injury; ligamentous dissection; anular disruption; retraction of vascular, visceral, and neural structures; and an increase in complications. ${ }^{15,16}$ These approaches can cause both short- and long-term pain and functional sequelae for the patient, with the posterior approach thought to be responsi-
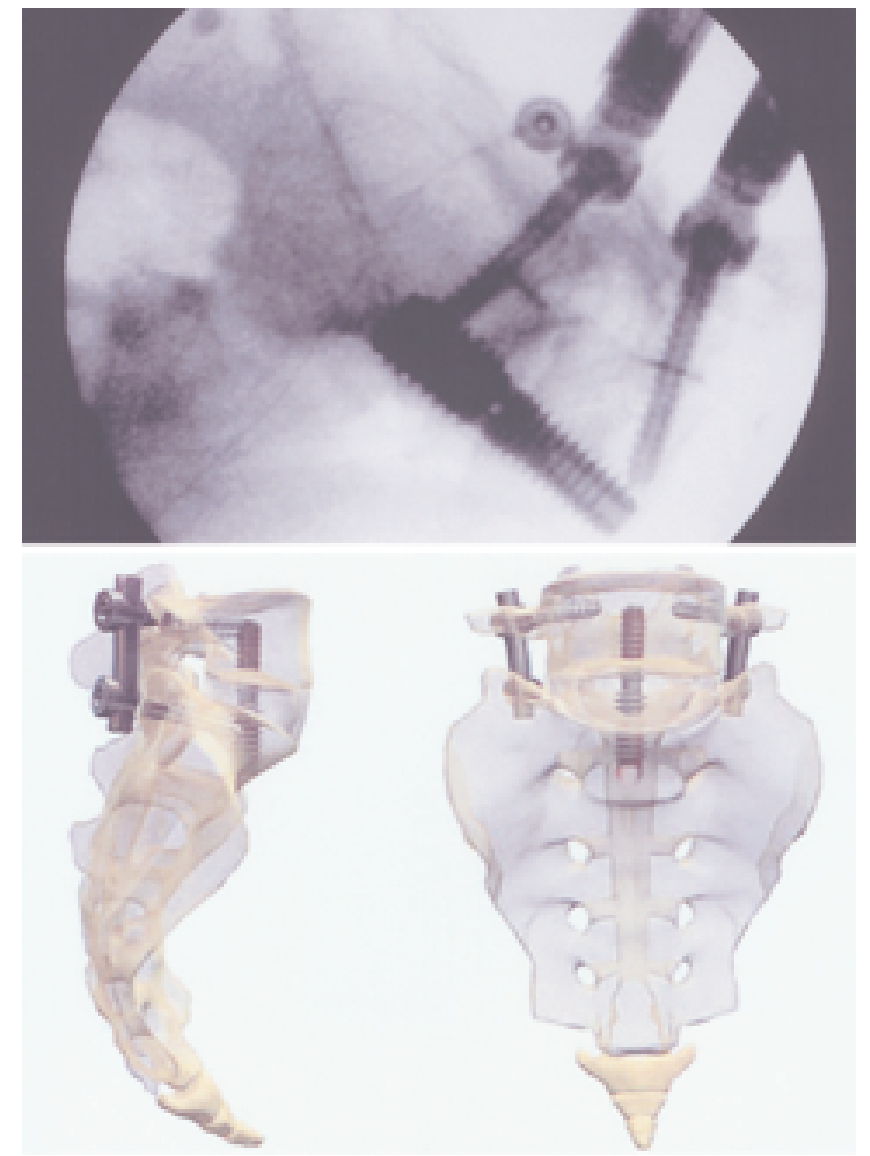

Fig. 3. Fluoroscopic image and illustrations. Upper and Lower: Using the Pathfinder minimally invasive percutaneous screws and extenders, supplemental pedicle fixation is completed across the L5-S1 space to prevent rotation and subsidence around the AxiaLif cage. The final construct is thus stiff in flexion, extension, lateral bending, shear, and translation.

ble for denervation of the dorsal spinal musculature in 10 to $20 \%$ of cases.

Whereas existing anterior approaches place the abdominal viscera, ureter, retroperitoneal structures, sympathetic plexi, and great vessels at risk, dorsal approaches instead cause significant harm to the posterior muscles, neural structures, and osseous stabilizing elements. Recently developed minimally invasive spinal surgical techniques now allow surgeons to perform interbody fusion and screw placement with less pain, damage to muscles, and blood loss; shorter postoperative length of stay; and fewer medications than open surgery. 7,8 To date, however, these minimally invasive posterior and anterior fusion techniques still require the use of a smaller version of the traditional open surgical corridors and thereby still place the same structures at risk. The development of this exciting, novel approach to the L5-S1 interbody space for the purposes of reconstruction, arthrodesis, and instrumentation represents a true departure from existing surgical approaches to this space. Through the naturally existing presacral fat pad, ready access can be gained to the disc space while completely avoiding the anterior abdominopelvic cavity, great vessels, neural elements, facets, lamina, and the dorsal musculoligamentous complex. 

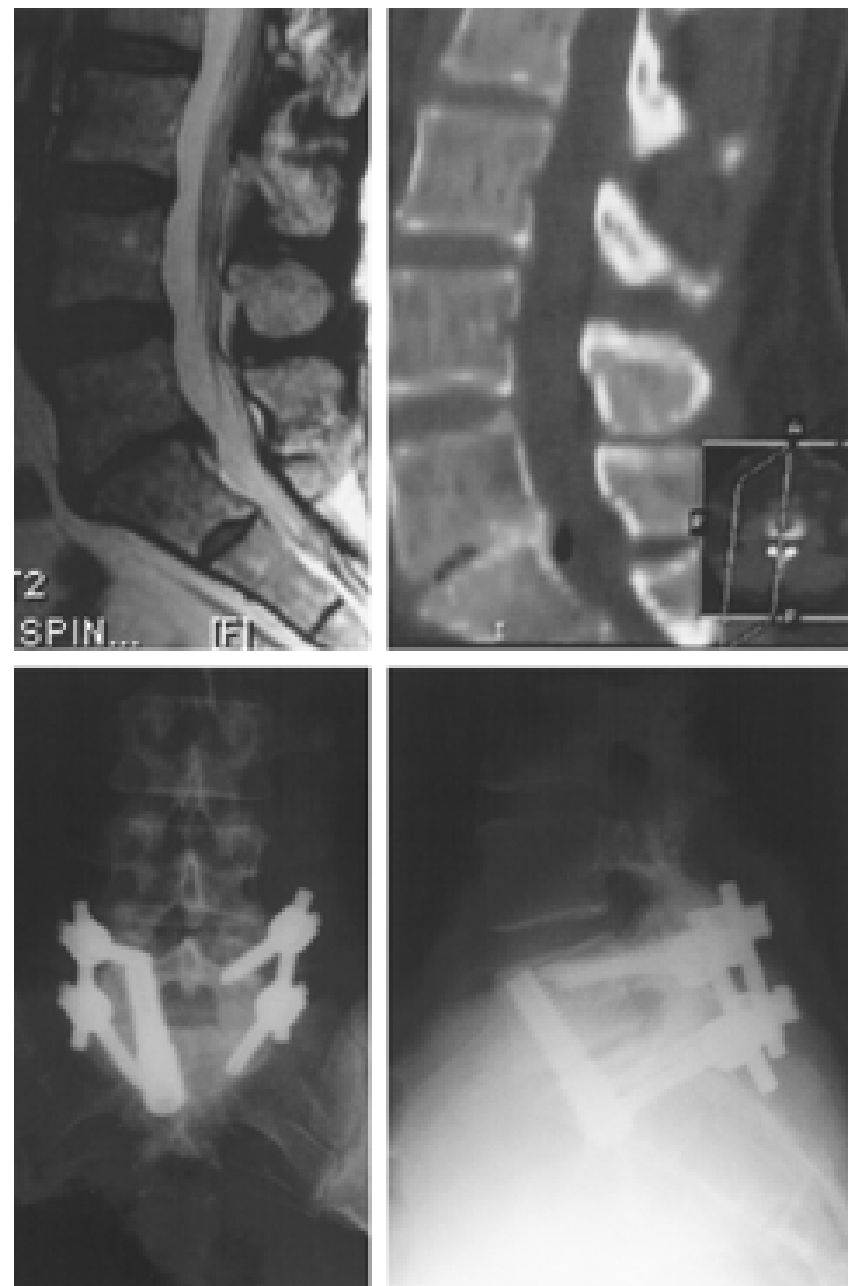

Fig. 4. Case 1. Upper: Preoperative sagittal MR images revealing the prior hemilaminotomy and the collapsed L5-S1 disc space, with significant loss of foraminal height and degenerative endplate changes. Lower: Postoperative AP (left) and lateral (right) radiographs demonstrating successful placement of the AxiaLif cage with supplemental percutaneous pedicle screws. Excellent restoration of the foraminal height is seen, with moderate intervertebral distraction.

The presacral space is bordered posteriorly by the parietal fascia that covers the sacrum and anteriorly by a visceral fascia commonly called the mesorectum. The presacral space is filled with organized connective tissue. Adipose tissue surrounds the rectum and is defined posteriorly by the mesorectum, which contains blood vessels supplying the rectum..$^{20}$ The neural structures in the presacral space are hypogastric nerves $1 \mathrm{~cm}$ lateral to the midline at the sacral promontory and parasympathetic nerves that arise from the ventral roots of S3-4 in men and S2-4 in women. At S1-2, the hypogastric nerves are located several centimeters laterally, so they are not at significant risk from a trajectory on the midline of the sacrum.

The width of the presacral space was measured retrospectively by using sagittal $\mathrm{T}_{2}$-weighted MR images obtained in 193 patients ( 87 men and 106 women, age range 18-83 years). The presacral space width was measured separately for the S-1, S-2, and S-3 vertebral levels from
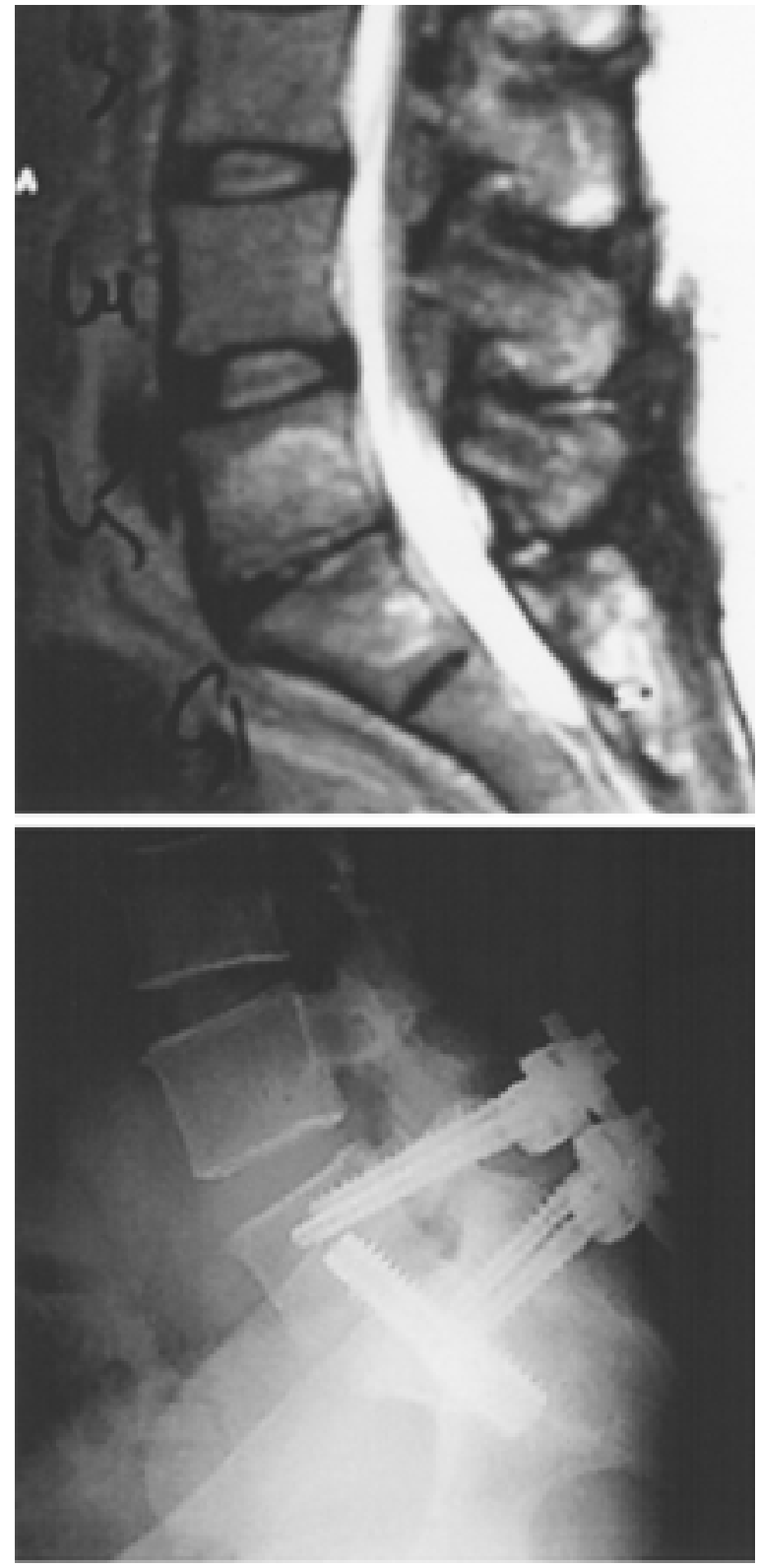

Fig. 5. Case 2. Upper: Preoperative sagittal MR image demonstrating Modic changes across the L5-S1 disc space, with significant loss of height and secondary foraminal stenosis. Lower: Postoperative lateral radiograph showing placement of the AxiaLif cage and screws, with early bridging bone seen in the space at 9 months.

the anterior surface of the VBs to the closest part of the posterior wall of the rectum. Differences between male and female individuals were analyzed using t-tests. Normal mean widths of the presacral space in men and women were 16.2 and $11.9 \mathrm{~mm}$ for $\mathrm{S}-1,14.9$ and $11.2 \mathrm{~mm}$ for $\mathrm{S}-2$, and 13 and $10.6 \mathrm{~mm}$ for S-3, respectively (Fig. 6). ${ }^{14}$ In the presacral space the vascular structures consist of the middle sacral artery and veins; these vessels are of variable size and are typically located in the region of the sacral promontory, which is more rostral than the S1-2 junction where the working cannulas and pins are docked. Bleeding from the 

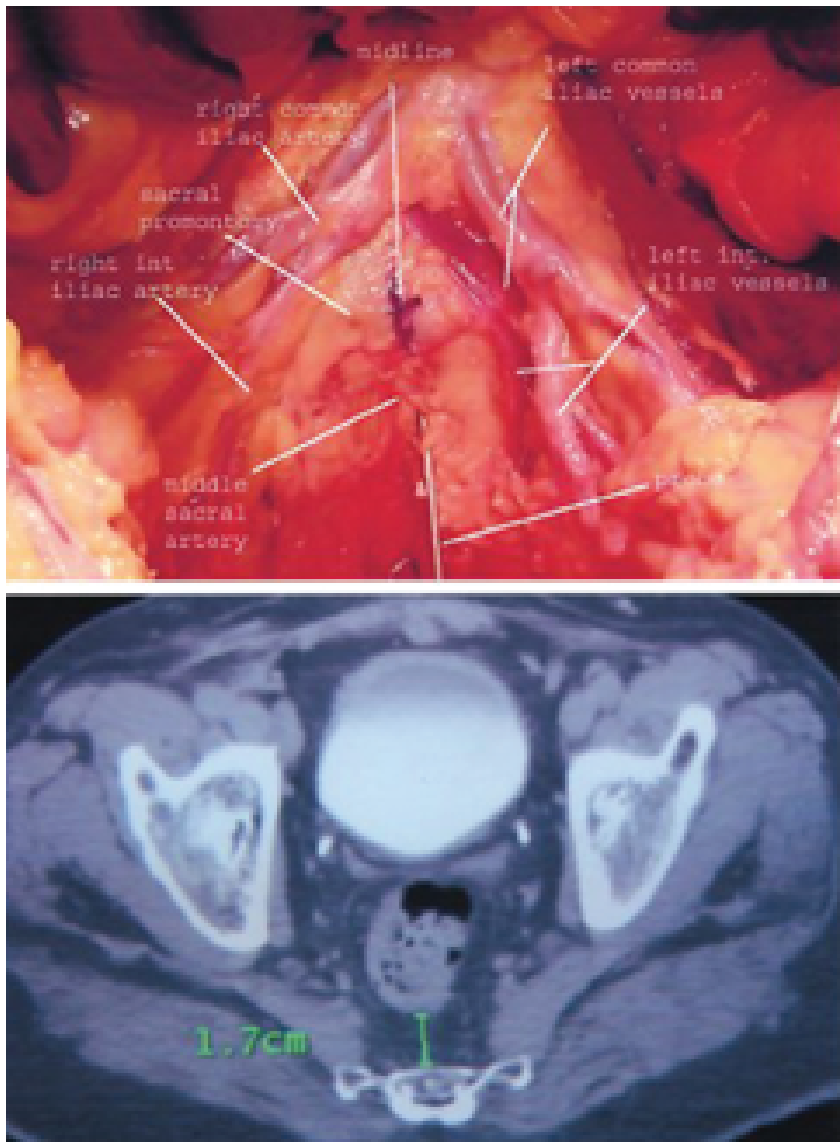

Fig. 6. Upper: Intraoperative photograph of the presacral space revealing the great vessels well lateral to the working area of the introducer stylet. The middle sacral artery lies within the working zone but is often vestigial, thereby allowing for blunt dissection by sweeping with the blunt tip of the trocar. Lower: Pelvic computerized tomography scan revealing a thick presacral working space. This space is typically 1.1 to $2 \mathrm{~cm}$ thick. (Oto A, Peynircioglu B, Eryilmaz M, et al: Determination of the width of the presacral space on magnetic imaging. Clin Anat 17:14-16, 2004) Int $=$ interval.

transverse sacral vein or the midline sacral artery is also possible, but at the $\mathrm{S}-2$ the midline sacral artery is often small or nonexistent. The risk of venous bleeding is low, as the technique begins with sweeping the soft tissues off the sacrum with a blunt dilator. The S1-2 junction thus represents an anatomically safer working zone with regard to neural and vascular injury. We believe the risk of infection related to the paracoccygeal approach has been low because the procedure is brief and percutaneous and results in very little devitalized tissue and anatomical dead space. To date, there have been no infectious complications in the more than 50 transsacral fusions that have been performed in North and South America, as reported by the parent company (TranS1, Inc.).

All anterior or posterior approaches for L5-S1 interbody arthrodesis, discectomy, endplate preparation, and grafting result in anular disruption and injury to either the anterior or posterior longitudinal ligament, with resulting biomechanical destabilization. ${ }^{3}$ With this novel paracoccygeal, transsacral approach to the L5-S1 interspace, surgeons can now perform a near-total discectomy without violating the anulus or surrounding ligaments, thereby significantly increasing the stiffness of the motion segment with distraction. In addition, this leaves the area around the disc, great vessels, and neural elements untouched and thus free of surgical scarring, which is attractive for future cases that may require revision or adjacent level surgery.

With the AxiaLif cage construct, significant segmental stiffness is immediately afforded by distraction across the disc space. As the ligaments and anulus are completely intact, this provides the strongest possible ligamentotaxis, thereby affording the best interbody fusion construct stiffness. There is also no need for retraction of the vascular or neural elements to place the prosthesis, because the size is limited only by the diameter of the working portal. As distraction is achieved by the differential screw pitch of the ends of the cage, a wide variety of distraction heights can be obtained by simply altering the design of the implant. When combined with percutaneous pedicle screw instrumentation such as the Pathfinder system, additional distraction, compression, and reduction manuvers can also be applied to the spine as needed prior to threading the cage across the interspace. Akin to transsacral fibular dowel struts in the stabilization of high-grade isthmic spondylolisthesis, axially placed cages such as the AxiaLif transsacral cage provide excellent resistance to shear, translation, flexion, and extension that is far superior to that of traditional interbody constructs. ${ }^{5,18}$

\section{CONCLUSIONS}

The axial approach to the lumbosacral spine is simple and reproducible. It is a novel technique that allows surgeons to perform an anterior interbody L5-S1 fusion with minimal risk to adjacent vital structures and without anular disruption, thereby increasing the immediate construct stiffness and providing the best milieu for subsequent successful fusion. Although long-term fusion and functional outcome data are required to ultimately validate this technique, early results have been extremely promising. In the future, this unique approach may also provide an exciting means not only of achieving multisegmental L4-5 and L5-S1 fusions, but also providing a corridor by which arthroplasty-type devices may be implanted while completely maintaining the musculoligamentous integrity, and therefore the stability, of the motion segment.

\section{Disclosure}

Drs. Khoo and Pimenta haveresearch grants from TranS1, Inc., and members of that company speakers bureau.

\section{References}

1. Cloyd DW, Obenchain TG: Laparoscopic lumbar discectomy. Semin Laparosc Surg 3:95-102, 1996

2. Cragg A, Carl A, Casteneda F, et al: New percutaneous access method for minimally invasive anterior lumbosacral surgery. J Spinal Disord Tech 17:21-28, 2004

3. Ethier DB, Cain JE, Yaszemski MJ, et al: The influence of anulotomy selection on disc competence. A radiographic, biomechanical, and histologic analysis. Spine 19:2071-2076, 1994 
4. Guiot BH, Khoo LT, Fessler RG: A minimally invasive technique for decompression of lumbar spine. Spine 27:432-438, 2002

5. Hanson DS, Bridwell KH, Rhee JM, et al: Dowel fibular strut grafts for high-grade dysplastic isthmic spondylolisthesis. Spine 27:1982-1988, 2002

6. Heniford BT, Mathews BD, Lieberman IH: Laparoscopic lumbar interbody fusion. Surg Clin North Am 80:1487-1500, 2000

7. Khoo LT, Fessler RG: Microendoscopic decompressive laminotomy for the treatment of lumbar stenosis. Neurosurgery 51 (5 Suppl):S146-S154, 2002

8. Khoo LT, Palmer S, Laich DT, et al: Minimally invasive percutaneous posterior lumbar interbody fusion. Neurosurgery 51 (5 Suppl):S166-S181, 2002

9. Leu HF, Hauser RK: Percutaneous endoscopic lumbar spine fusion. Neurosurg Clin North Am 7:107-117, 1996

10. MacMillan M, Fessler RG, Gillepsy M, et al: Percutaneous lumbosacral fixtion and fusion: anatomic study and two-year experience with a new method. Neurosurg Clin N Am 7:99-106, 1996

11. Mathews HH: Percutaneous interbody fusion. Orthop Clin North Am 29:647-653, 1998

12. Mayer HM: The ALIF concept. Eur Spine J 9 (Suppl 1): S35-S43, 2000

13. Muhlbauer M, Pfisterer W, Eyb R, et al: Minimally invasive retroperitoneal approach for lumbar corpectomy and anterior reconstruction. Technical note. J Neurosurg 93 (1 Suppl): $161-167,2000$
14. Oto A, Peynircioglu B, Eryilmaz M, et al: Determination of the width of the presacral space on magnetic imaging. Clin Anat 17:14-16, 2004

15. Perez-Cruet MJ, Fessler RG, Perin NI: Review. Complications of minimally invasive spinal surgery. Neurosurgery 51 (5 Suppl):S26-S36, 2002

16. Rajaraman V, Vingan $\mathrm{R}$, Roth $\mathrm{P}$, et al: Visceral and vascular complications resulting from anterior lumbar interbody fusion. J Neurosurg 91 (1 Suppl):60-64, 1999

17. Regan JJ, Yuan H, McAfee PC: Laparascopic fusion of the lumbar spine: minimally invasive spine surgery. A prospective multicenter study evaluating open and laparoscopic lumbar fusion. Spine 24:402-411, 1999

18. Slosar PJ, Reynolds JB, Koestler M: The axial cage. A pilot study for interbody fusion in a higher-grade spondylolisthesis. Spine J 1:115-120, 2001

19. Trambert JJ: Percutaneous interventions in the presacral space: CT guided precoccygeal approach-early experience. Radiology 213:901-904, 1999

Manuscript received August 10, 2005.

Accepted in final form September 1, 2005

Address reprint requests to: Larry T. Khoo, M.D., 1245 16th Street, Suite 220, Santa Monica, California 90404, email: 1khoo@ mednet.ucla.edu. 